\title{
Kilohertz QPOs in low-mass X-ray binaries as oscillation modes of tori around neutron stars - I
}

\author{
Marcio G. B. de Avellar, ${ }^{1,2 \star}$ Oliver Porth, ${ }^{2}$ Ziri Younsi ${ }^{2}$ and Luciano Rezzolla ${ }^{2,3}$ \\ ${ }^{1}$ Instituto de Astronomia, Geofísica e Ciências Atmosféricas, Universidade de São Paulo, 05508-090 São Paulo, Brazil \\ ${ }^{2}$ Institut für Theoretische Physik, Johann Wolfgang Goethe-Universität, Max-von-Laue-Str. 1, D-60438 Frankfurt am Main, Germany \\ ${ }^{3}$ Frankfurt Institute for Advanced Studies, Ruth-Moufang-Strasse 1, D-60438 Frankfurt am Main, Germany
}

Accepted 2017 November 24. Received 2017 November 10; in original form 2017 September 11

\begin{abstract}
There have been many efforts to explain the dynamical mechanisms behind the phenomenology of quasi-periodic oscillations (QPOs) seen in the X-ray light curves of low-mass X-ray binaries. Up to now, none of the models can successfully explain all the frequencies observed in the power spectral density of the light curves. After performing several general-relativistic hydrodynamic simulations of non-self-gravitating axisymmetric thick tori with constant specific angular momentum oscillating around a neutron star such as the one associated with the low-mass X-ray binary $4 \mathrm{U} 1636$ - 53, we find that the oscillation modes give rise to QPOs similar to those seen in the observational data. In particular, when matching pairs of kilohertz QPOs from the numerical simulations with those observed, certain combinations reproduce well the observations, provided we take a mass for the neutron star that is smaller than what is generally assumed. At the same time, we find that tori with constant specific angular momentum cannot match the entire range of frequencies observed for $4 \mathrm{U} 1636-53$ due to physical constraints set on their size. Finally, we show that our results are consistent with the observed shifts in QPO frequency that could accompany state transitions of the accretion disc.
\end{abstract}

Key words: accretion, accretion discs - stars: oscillations - X-rays: binaries.

\section{INTRODUCTION}

Several X-ray variability components can be seen in the power spectral density (PSD) of the X-ray light curves of neutron star and black hole low-mass X-ray binaries (NS and BH LMXBs, respectively). Among other components, there is a vast set of quasi-periodic oscillations (QPOs) ranging from $\sim 0.01$ to $\sim 1000 \mathrm{~Hz}$, whose physical origin (origins) is still an open problem (see van der Klis 2006, for a comprehensive review of QPOs in LMXBs). Overall, there are two broad classes of models that address the mechanism behind the QPOs with different degrees of success. The first one relies on orbital motions (including the general-relativistic epicyclic motions) of matter around the compact object and their time-scales (see, for example, Miller, Lamb \& Psaltis 1998; Stella \& Vietri 1999; Abramowicz et al. 2004; Motta et al. 2014). The second class, the focus of this study, interprets the QPOs as oscillations and/or flow instabilities of some kind of an accretion disc orbiting around the central object (see, for example, Rezzolla et al. 2003b; Fragile, Straub \& Blaes 2016). The study of accretion flows around compact objects dates back to the 1970s with the pioneering works of Shakura \& Sunyaev (1973) and Novikov \& Thorne (1973) when considering geometrically thin accretion discs, and to the works of Fishbone \& Moncrief (1976), Abramowicz, Jaroszynski \& Sikora (1978), and Kozlowski, Jaroszynski \& Abramowicz (1978) in the opposite case of geometrically thick discs. The latter are possible solutions of a stationary and axisymmetric fluid that undergoes a non-Keplerian circular motion around a compact object. Despite the variety of mechanisms proposed so far, the scarcity of the observational data and the weak constraints it poses do not seem to favour one model over the other.

Astronomical observations over the years have revealed that the frequencies of different QPOs observed in a given source correlate with each other through very specific patterns and, more importantly, that the same patterns are then seen across systems of the same type (e.g. van Straaten et al. 2002; van Straaten, van der Klis \& Méndez 2003; Reig, van Straaten \& van der Klis 2004; van Straaten, van der Klis \& Wijnands 2005; Altamirano et al. 2008; Motta et al. 2014, 2015). The correlations seen in the PSDs of different types of X-ray binaries, i.e. containing BHs, NSs or white dwarfs, seem to indicate that a common component of these systems, most likely the accretion disc, is responsible for the origin of these variability features (e.g. Wijnands \& van der Klis 1999; Psaltis, Belloni \& van der Klis 1999; Mauche 2002; Warner \& Woudt 2002; Motta et al. 2017). Within this accretion-disc interpretation, the highest frequency QPOs, known as the lower $\left(v_{l}\right)$ and upper $\left(v_{u}\right)$ kilohertz 
QPOs (kHz QPOs), are thought to reflect the properties of the accretion flow in the vicinity of BHs and NSs, where effects of the general relativity are expected (see van der Klis 2006).

Other properties of the QPOs, besides the frequency itself, like the fractional amplitude, quality factor, and time/phase lags, also depend on the spectral state of the source, which can be parametrized by the position of the source in what is called the "colour-colour diagram' (CCD) in X-rays (e.g. Hasinger \& van der Klis 1989; Wijnands et al. 1997; van Straaten et al. 2002; Di Salvo, Méndez \& van der Klis 2003; Méndez 2006; Altamirano et al. 2008; de Avellar et al. 2016). The changes in the position of the source within the $\mathrm{CCD}$ are therefore expected to reflect, at least qualitatively, different configurations of the flow near the central compact object (see Altamirano et al. 2008, for example).

From the theoretical point of view, geometrically thick discs achieved astrophysical relevance once it was demonstrated that external perturbations cause them to oscillate periodically, opening new avenues for the interpretation of the phenomenology of QPOs (e.g. Abramowicz et al. 2003, 2004; Rezzolla, Yoshida \& Zanotti 2003a; Rezzolla et al. 2003b; Zanotti, Rezzolla \& Font 2003; Bursa et al. 2004; Kluźniak et al. 2004; Lee, Abramowicz \& Kluźniak 2004; Zanotti et al. 2005; Blaes, Arras \& Fragile 2006; Remillard \& McClintock 2006; Schnittman \& Rezzolla 2006; Blaes et al. 2007; Montero \& Zanotti 2012; Mazur et al. 2013; Vincent et al. 2014; Bakala et al. 2015; Mishra et al. 2017, and references therein for the vast literature that has developed over the years). In particular, Rezzolla et al. (2003a) identified the frequencies in the X-ray spectra of BH LMXBs as inertial-acoustic modes, or $p$ modes, of a relativistic geometrically thick toroidal accretion disc. The model is successful in reproducing approximately the $3: 2$ ratio seen in the range of the high-frequency (HF) QPOs observed in these kinds of systems. Further investigations of the properties of axisymmetric $p$-mode oscillations were later on performed by, for example, Zanotti et al. (2003, 2005), Montero et al. (2007), Montero, Font \& Shibata (2008) and Montero, Font \& Shibata (2010) through general-relativistic hydrodynamical (GRHD) simulations of both non-self-gravitating and self-gravitating tori around compact objects.

This paper extends these simulations to NS LMXBs, with particular application to the Atoll ${ }^{1}$ source 4U 1636-53, for which more than $15 \mathrm{yr}$ of X-ray data have been collected and analysed in their many aspects (see, for example, Di Salvo et al. 2003; Barret, Olive \& Miller 2006; Casares et al. 2006; Altamirano et al. 2008; Lin et al. 2011; Sanna et al. 2012; Artigue et al. 2013; de Avellar et al. 2013; Lyu, Méndez \& Altamirano 2014; Sanna et al. 2014; de Avellar et al. 2016; Ludlam et al. 2017; Zhang et al. 2017, for an incomplete set of references, see references therein). More specifically, this paper details (i) how we build a sequence of 21 relativistic and axisymmetric tori with constant specific angular momentum and follow their oscillations triggered by a small radial and vertical velocity perturbation, and (ii) how an observational data set analysed by one of us (de Avellar et al. 2013, 2016) is used to constrain the model.

The plan of the paper is as follows. In Section 2.3, we discuss how frequencies are identified from the simulation data and crosscompared to the observational $v_{l}-v_{u}$ relation (Section 3). The results are discussed in Section 4, elucidating the implications of our analysis with application to the changing properties of the $\mathrm{kHz}$

\footnotetext{
${ }^{1}$ For a description of the $\mathrm{Z}$ and Atoll sources, see Hasinger \& van der Klis (1989).
}

QPOs as the source moves across the CCD. Throughout we adopt the geometrized system of units $G=c=1$, with $G$ Newton's constant and $c$ the speed of light.

\section{NUMERICAL SETUP}

\subsection{Initial configurations of the tori}

As a first step we describe the construction of the initial equilibrium torus solutions of which the oscillation frequencies are analysed in the remainder of this paper. We consider tori as stationary non-selfgravitating fluid configurations that can be built around a compact object as a solution for the GRHD equations (as in Kozlowski et al. 1978, for example). To simulate the dynamics of this system, we solve the usual GRHD equations written in their covariant form

$\nabla_{\mu}\left(\rho u^{\mu}\right)=0$

$\nabla_{\mu} T^{\mu v}=0$,

where $\rho$ is the rest-mass density, $u^{\mu}$ are the (contravariant) components of the fluid four-velocity, and $T^{\mu v}$ is the energy-momentum tensor (see, for example, Rezzolla \& Zanotti 2013).

To close the system of equations (1) and (2), we assume an ideal-fluid equation of state (Rezzolla \& Zanotti 2013) $p=\rho \epsilon(\hat{\gamma}-$ 1 ), where $\epsilon$ is the specific internal energy and $\hat{\gamma}$ is the adiabatic index. The initial (test-fluid) equilibrium is obtained as isentropic configuration; thus, we set the entropy $\kappa=p / \rho^{\hat{\gamma}}$ to an arbitrary constant (we have taken $\kappa=0.001$ ). ${ }^{2}$ As a result, the specific enthalpy $h(\rho, p)$ is expressed as

$h=1+\epsilon+\frac{p}{\rho}=1+\frac{\hat{\gamma}}{\hat{\gamma}-1} \frac{p}{\rho}$.

Since the fluid is following a non-Keplerian motion, the distribution of specific angular momentum $\ell:=u_{\phi} / u_{t}$ needs to be specified, where $u_{\phi}$ and $u_{t}$ are the azimuthal and time components of the fourvelocity, respectively. The simplest choice in this case, and the one that has been historically best studied, is to set $\ell=$ const., so that the maximum equatorial torus size for the marginally stable nonaccreting configuration is determined by the value of the specific angular momentum distribution for the fluid (Font \& Daigne 2002; Daigne \& Font 2004; Rezzolla \& Zanotti 2013).

As mentioned in Section 1, we use the NS LMXB in 4U 1636 - 53 as our study case, and the torus should therefore not penetrate the surface of the star, assumed to have a mass which falls in the uncertainty bracket suggested by Casares et al. (2006), i.e. $M=1.7 \mathrm{M}_{\odot}$. Assuming the representative nuclear-physics equation of state SLy4 of Douchin \& Haensel (2001) to such a mass would correspond to a NS with radius of $11.42 \mathrm{~km}$ when non-rotating.

Since the exterior space-time is given by the Schwarzschild solution with $M$ the mass of the neutron star, we choose values of the constant specific angular momentum $\ell \in[3.68,3.81]$, noting that a torus with $\ell=3 \sqrt{6} / 2 \simeq 3.67$ is actually a ring (Rezzolla $\&$ Zanotti 2013). We recall, in fact, that the position of the cusp and the centre, which are the location where the specific angular momentum coincides with the corresponding Keplerian one, are both at $6 M$ for a non-rotating central star of mass $M$, and the orbital frequency of the fluid in this case tends to the orbital frequency of a test particle.

\footnotetext{
${ }^{2}$ We recall that ideal-fluid and the polytropic equations of state coincide for isentropic transformations as it is the case for the initial data (Rezzolla \& Zanotti 2013).
} 
Table 1. Parameters of the models built and simulated in this work. Reported are the resolution, specific angular momentum $\left(\ell_{o}\right)$, the position of the cusp $\left(r_{\text {cusp }}\right)$, the position of the inner edge of the torus $\left(r_{\text {in }}\right)$, the position of the centre of the torus $\left(r_{\text {centre }}\right)$, the outer edge of the torus $\left(r_{\text {out }}\right)$, and the size of the torus; for each quantity, the corresponding units are indicated in parentheses. Note that only the odd-numbered models from Tor. 03 to Tor. 21 have been simulated numerically.

\begin{tabular}{lccccccc}
\hline Models & Resolution & $\ell_{o}\left(M^{2}\right)$ & $r_{\text {cusp }}(\mathrm{M})$ & $r_{\text {in }}(M)$ & $r_{\text {centre }}(M)$ & $r_{\text {out }}(M)$ & size $(\mathrm{km})^{a}$ \\
\hline Tor.03 & $2624 \times 1728^{b}$ & 3.6932 & 5.3607 & 5.5617 & 6.7774 & 7.8297 & 5.69 \\
Tor.05 & $1920 \times 1280^{b}$ & 3.7064 & 5.1908 & 5.4529 & 7.0437 & 8.6271 & 7.97 \\
Tor.07 & $1472 \times 960^{b}$ & 3.7196 & 5.0606 & 5.3693 & 7.2707 & 9.4006 & 10.12 \\
Tor.09 & $1216 \times 832^{b}$ & 3.7328 & 4.9533 & 5.2951 & 7.4753 & 10.1923 & 12.29 \\
Tor.11 & $1040 \times 700$ & 3.7460 & 4.8612 & 5.2306 & 7.6649 & 11.0196 & 14.53 \\
Tor.13 & $900 \times 600$ & 3.7592 & 4.7801 & 5.1721 & 7.8439 & 11.9001 & 16.89 \\
Tor.15 & $780 \times 520$ & 3.7724 & 4.7075 & 5.1170 & 8.0148 & 12.8531 & 19.42 \\
Tor.17 & $680 \times 460$ & 3.7856 & 4.6416 & 5.0575 & 8.1793 & 13.9106 & 22.22 \\
Tor.19 & $600 \times 400$ & 3.7988 & 4.5812 & 5.0073 & 8.3386 & 15.0653 & 25.25 \\
Tor.21 & $560 \times 380$ & 3.8062 & 4.5494 & 4.9771 & 8.4262 & 15.7797 & 27.12 \\
\hline A & & & & & &
\end{tabular}

${ }^{a}$ Assuming a NS mass of $1.7 \mathrm{M}_{\odot}$.

${ }^{b}$ Resolution reached in the torus area after three levels of mesh refinement.

Hence, a torus with $\ell \geq 3.81$ would penetrate the stellar surface, i.e. it would have $r_{\text {cusp }}<r_{\text {star }}$.

Also, in order to minimize mass-loss at each oscillation, we employ a jump in the effective potential, $\Delta W:=W_{\text {in }}-W_{\text {cusp }}$, where $W_{\text {in }}$ is the potential at the inner edge of the torus and $W_{\text {cusp }}$ is the potential at the cusp of the of the potential well, specifying $W_{\text {in }}$ in a way that the size of the torus is $\simeq 90$ per cent of the maximum equatorial torus size for the marginally stable non-accreting configuration. Hence, the inner edge of each torus is always safely below the inner edge of the marginally stable one. Following the prescription described above and which resembles what suggested by Font \& Daigne (2002), we have built 21 axisymmetric torus models of increasing size; the main properties of some of these models are summarized in Table 1.

The oscillations are triggered by specifying small radial and vertical perturbations in the four-velocity field given by

$\left(\delta v^{i}\right)=\left(0, \frac{\eta}{\sqrt{2 g_{r r}}}, \frac{\eta}{\sqrt{2 g_{\theta \theta}}}, 0\right)$,

where $\eta:=0.01 \sqrt{W_{\text {in }}-W_{\text {centre }}}$ and $W_{\text {centre }}$ is the potential at the centre of the torus and which also corresponds with the position of the maximum rest-mass density, ensuring that the perturbation in the vertical and radial directions has the same magnitude as can be readily seen by computing the scalar product $\delta v^{i} \delta v_{i}=\eta^{2}$. Since the quantity $\sqrt{W_{\text {in }}-W_{\text {centre }}}$ is proportional to the 'escape velocity' from the potential well, the perturbation in the velocity is comparable across all torus models.

There are two reasons why we restricted the tori to have their cusp outside the star while not filling them with matter up to the whole potential well. On one hand, the cusp inside the star would let us to build a bigger torus, producing lower frequencies, which is interesting (see Section 3), but on the other hand, simulations showed that this would lead to a significant mass-loss at each oscillation cycle. The second reason is that we are dealing with accretion for subsequent papers of this series and the cusp being outside will be important.

\subsection{Numerical approach}

The simulations were carried out using the $\mathrm{BH}$ accretion code BHAC (Porth et al. 2017), which solves the ideal general-relativistic magnetohydrodynamic (GRMHD) equations in arbitrary space- times, exploiting shock-capturing techniques and adaptive mesh refinement. For a detailed description of the code and its capabilities, see Porth et al. (2017). BHAC is a versatile code that allows the user to freely choose the metric theory of gravity and coordinate system. Here, we use the Schwarzschild metric in modified KerrSchild coordinates, whose main purpose is to remove the coordinate singularity at the horizon but also to stretch the grid radially thus reducing the computational cost.

Our grid extends from the NS radius at $r_{\text {star }} \simeq 4.549 M$ to $30 M$ in the radial direction and from $\pi / 2-0.628$ to $\pi / 2+0.628$ in the $\theta$ direction. The resolution of the grid was chosen in order resolve all tori with $\simeq 200$ cells in their diameter. Since uniform resolution would have been impractical for simulations of small tori, three levels of mesh refinement were employed in the smallest tori (cf. Table 1).

\subsection{Adopted methodology for the data analysis}

Although we have constructed 21 different initial models representing stationary tori, our evolutions have been performed using only the odd-numbered models from Tor.03 to Tor.21, for a total of 10 models simulated. This choice was dictated by the need to reduce the computational costs but does not come at the cost of reducing the generality of our results and conclusions. Also, models smaller than Tor. 03 were not considered because they turn out too small to be physically meaningful. We recall, in fact, that as the torus' size goes to zero (both in the radial and in the polar directions), the corresponding oscillations approach those of a test particle at the position of the centre, which can be readily computed analytically (Rezzolla et al. 2003b; Zanotti, Rezzolla \& Font 2003).

We have therefore evolved each of the ten models up to $t=60000 \mathrm{M}$, which is equivalent to $\simeq 500$ orbital periods, and corresponds to a time-scale of $\sim 500 \mathrm{~ms}$ when assuming $M=1.7 \mathrm{M}_{\odot}$. Due to the velocity perturbation, the tori will oscillate inside the potential well, possibly leading to mass-loss or induced accretion at each period. Specifying $W_{\text {in }}$ as mentioned in Section 2.1 and as shown in Table 1, we minimize this effect. As a result, the oscillation can survive in all runs with only a minimal damping due to the mass-loss; no significant drift in frequencies is observed as a consequence of this prescription.

Rezzolla et al. (2003b) and Zanotti et al. (2003) studied toroidal fluid configurations around Schwarzschild BHs having a constant 

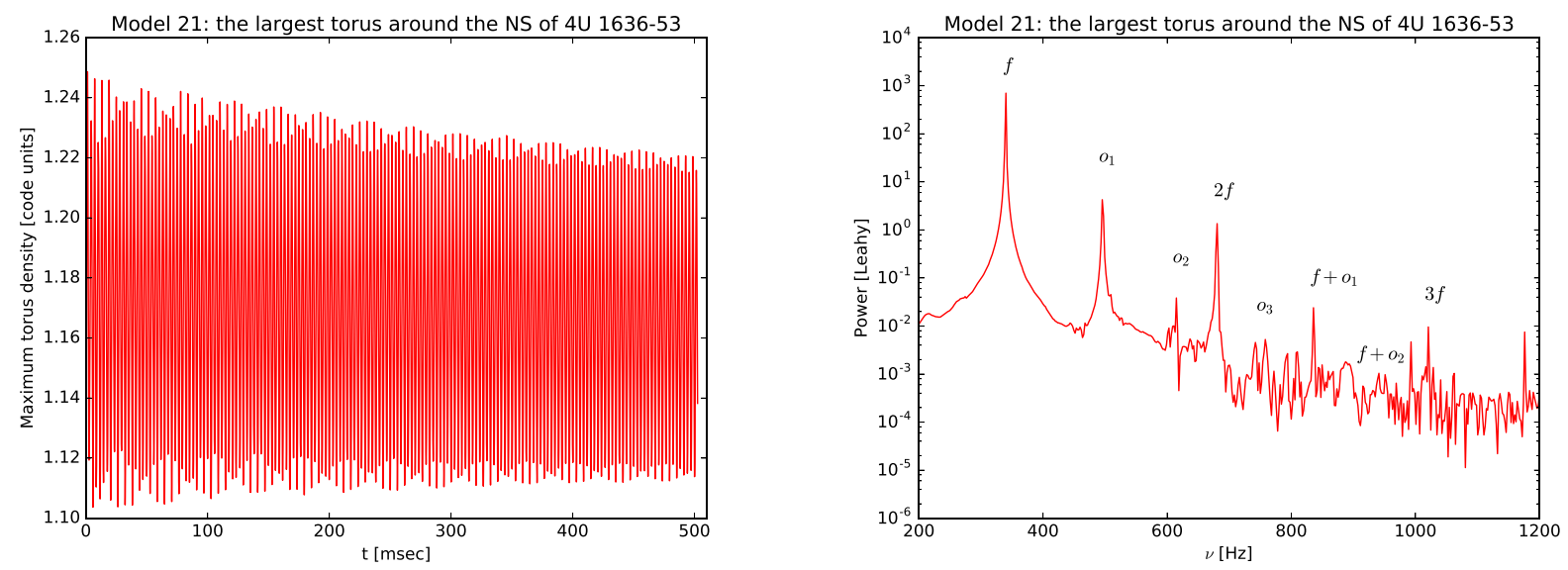

Figure 1. Oscillations in the maximum rest-mass density (left-hand panel) and its corresponding PSD (right-hand panel) of Tor.21, the largest torus around the NS in 4U 1636 - 53 (see Table 1). The small damping in the density is due to $\sim 1$ per cent mass-loss, which is also the largest measured in our simulations. Other tori show very similar behaviours, with the position and amplitude of the modes obviously depending on the specific torus considered.

specific angular momentum distribution; the analysis by Rezzolla et al. (2003b) was semi-analytical and involved linear perturbation theory, whereas the analysis by Zanotti et al. (2003) was fully numerical and involved general-relativistic hydrodynamic simulations. Notwithstanding the two different approaches, both analyses found that the $p$ modes of these configurations appear in frequencies obeying the $2: 3: 4: \ldots$ ratio. Zanotti et al. (2005), on the other hand, studied through both linear analysis and simulations, toroidal fluid configurations around Kerr BHs also having non-constant specific angular-momentum distributions; as a result, a richer set of frequencies was simulated and measured. The linear perturbative analysis formulated a prediction about the eigenfrequencies of the $p$ modes and the ratio in which they appear, thus providing a theoretical insight into where to look for the frequencies coming from the simulations. In either case, the authors studied the response of the $L_{2}$-norm of the density, which as a global quantity is particularly suitable for comparisons with the results coming from the perturbative analysis.

In our analysis, we have followed the periodic response of the central rest-mass density of the tori $\left(\rho_{\max }\right)$ to the velocity perturbation. The choice for the central density is physically motivated in the sense that it is where the concentration of matter is larger and where most likely the variations in (X-ray) luminosity occur, i.e. where the QPOs would come from (see Schnittman \& Rezzolla 2006; Vincent et al. 2014).

We note that the responses of a given hydrodynamical quantity, e.g. the central rest-mass density and of its $L_{2}$-norm over the computational domain $\Omega$, i.e. $L_{2}(\rho):=\sqrt{\int_{\Omega} \rho^{2} \mathrm{~d} V / \int_{\Omega} \mathrm{d} V}$, should be equivalent. However, it was found that while the frequency of the peaks remains the same for both quantities, the strength of the peaks and even their appearance depend to varying degree on the quantity under consideration. This fact raises the question of how to appropriately identify the peaks to be considered in the analysis, and as a consequence a suitable strategy was developed for this purpose (see discussion in Section 2.3.1). Fig. 1 reports the oscillations of the central rest-mass density $\rho_{\max }$ and its corresponding PSD for Tor.21, which the largest torus around the NS in $4 \mathrm{U} 1636$ - 53. The mass lost in this case is the largest of all the models considered, but it is only of the order of 1 per cent.

\subsubsection{Frequency identification}

In order to proceed with the identification of the modes observed, we have followed the linear analysis by Rezzolla et al. (2003b); Zanotti et al. (2003) and Zanotti et al. (2005), and first identified and labelled the $p$-mode fundamental frequency and its overtones using the same nomenclature, as shown in the right-hand panel of Fig. 1 for the representative torus model Tor.21. Proceeding in this manner, we were able to determine eight different frequencies of increasing value: $f, o_{1}, o_{2}, 2 f, o_{3}, f+o_{1}, f+o_{2}$, and $3 f$. These peaks in the PSD correspond to the same frequencies identified by Zanotti et al. (2005) in their analysis, with the potential addition of the third overtone labelled $o_{3}$, whose excess power is however rather small.

However, we recall that not all frequencies are detected in all models. Additional tests were performed to ensure that in this study of the QPOs only reliable peaks were used. More information can be gained by logarithmically binning our data and comparing the binned PSDs with the non-binned ones. ${ }^{3}$ Logarithmically rebinning the data and averaging the same number of points per decade result in a rebinning factor of $10^{1 / N}$. Performing the binning correctly will add power to a feature which is present in the data while smearing out the noise. Hence, we have adopted two binning factors to determine whether a given peak was genuine of the possible contamination from noise: $N=200$ (infrequent binning) and $N=100$ (frequent binning). An example of this procedure is illustrated in Fig. 2 for Tor. 07.

In this way, a systematic study of the statistics of the various models was performed through which we counted how often a given peak was detected 'robustly' through all simulation models. By 'robust', we here refer to a peak that needed to be detected for at least seven torus models and both in the PSD of the maximum rest-mass density and in the PSD of the corresponding $L_{2}$-norm. This validation was necessary to obtain the confidence that the peak considered actually referred to a physical mode of oscillation. Following these criteria for the identification of the peaks in the PSD and for the determination of their robustness, the following frequencies were ultimately selected: $f, o_{1}, o_{2}, 2 f, o_{3}$, and $f+o_{1}$.

\footnotetext{
${ }^{3}$ Rebinning by a factor $N$ means that $N$ points are taken and averaged into a single value.
} 


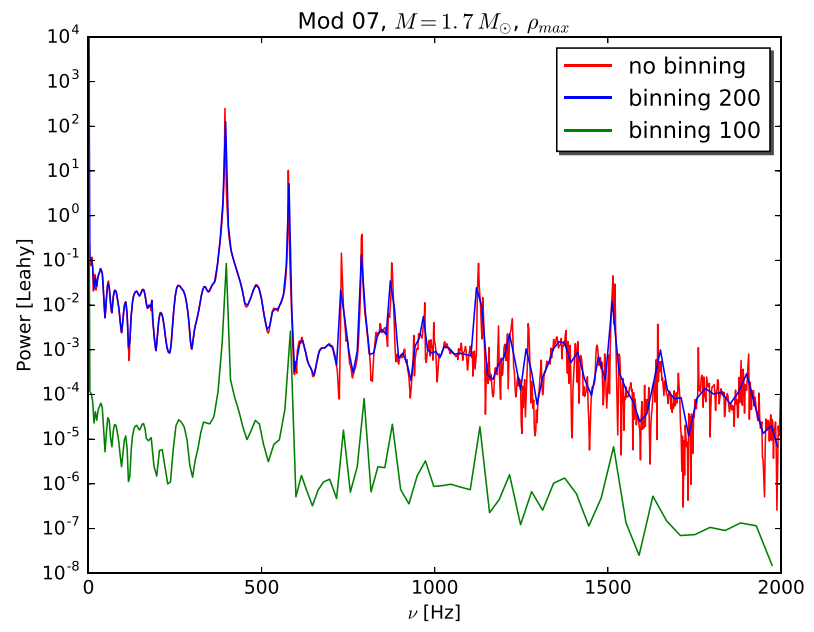

Figure 2. Examples of binned and not binned PSDs of the maximum restmass density. The data refer to the model Tor. 21 and show that even a low binning rate retains the main features of the PSDs.

The peaks associated with the frequencies $f+o_{2}$ and $3 f$ did not pass these tests and were therefore not considered in the subsequent analysis, even though they appear clearly in some models.

In view of recent theoretical developments, it is worth mentioning that the $f$ mode corresponds to the radial epicyclic mode and the $o_{1}$ mode corresponds to the plus mode (see Blaes et al. 2006; Mishra et al. 2017). Other modes calculated by these authors are a bit more cumbersome to identify. We talk more about this later.

\section{RESULTS AND DISCUSSION}

A first and possibly most important result of our analysis is that the most prominent peaks of the oscillation modes of tori around NSs obey specific ratios, as shown in Table 2, and in agreement with the linear analysis performed by Rezzolla et al. (2003a) and by Zanotti et al. (2003). A summarizing representation of this conclusion is presented in Fig. 3, where we show the observational $v_{l} \times v_{u}$ relation (red dots with errorbars) for the NS LMXB 4U 1636 - 53, together with some choices of pairs of peaks (the frequencies of the modes of oscillation) produced by the simulated oscillating tori. The observational data used here come from the analysis presented in de Avellar et al. $(2013,2016)$ in the context of time/phase lags and the CCD, which will be important in the next papers of this series when we couple the general-relativistic radiative transfer. In the following discussion, we will concentrate on two specific cases, as these are the most representative and interesting.
We note that it is possible that no special relation actually exists between $v_{l}$ and $v_{u}$ (see Belloni, Méndez \& Homan 2005, 2007). Hereafter, we will use self-consistent general-relativistic calculations to determine whether or not these relations exist and, if they do exist, whether they can explain the observations relative to $4 \mathrm{U}$ $1636-53$.

The chosen frequency pairs reported in Fig. 3 are meant to match the lower $\left(v_{l}\right)$ and the upper $\left(v_{u}\right) \mathrm{kHz}$ QPOs. Red dots represent the observed data, whereas the points with colours different from red represent the frequencies measured from our simulated tori. Clearly, all of the simulated data sets show a remarkably linear relation between the chosen frequencies. In addition, we have also found that there is a non-linear relation between the torus size and oscillation frequency with an overall deviation of $\sim 10$ per cent over all considered models. As shown in Fig. 4, larger tori display in general smaller frequencies. However, below a critical torus size of $\simeq 12 \mathrm{~km}$ (for a stellar mass of $1.4 \mathrm{M}_{\odot}$ ), all frequencies start to decrease as the size of the torus is decreased.

Since the oscillation frequencies are related to the typical timescales associated with the central object, and thanks to the simplicity of the Schwarzschild metric which we use for the exterior of the relativistic star, the only degree of freedom is the stellar mass $M$. As a result, all of the reported frequencies can be scaled simply as $v_{1} / v_{2}=M_{2} / M_{1}$. Stated differently, decreasing the mass of the NS shifts the peaks to higher frequencies. However, although the mass of the compact object does not change the ratio between the peaks, i.e. $v_{u} / v_{l}$, it affects the peak separation between these two peaks in the same ratio $M_{2} / M_{1}$. This is behaviour is clearly shown by the dual representation in the two panels of Fig. 3, where the left-hand panel shows the effect of assuming a NS mass of $M=1.7 \mathrm{M}_{\odot}$. The right-hand panel, on the other hand, reports the same data and the corresponding correlations when the mass of the star is assumed to be $M=1.4 \mathrm{M}_{\odot}$. In this case, one can see that three choices of pairs of peaks provide all rather good representations of observational relation: peaks $o_{1}$ and $2 f$ (black upside down triangles), peaks $o_{1}$ and $o_{3}$ (green diamonds), and peaks $o_{2}$ and $f+o_{1}$ (blue squares). At the same time, we should recall that this value of the mass for the NS is smaller of what estimated by Casares et al. (2006), who have constrained the mass of the central compact object in 4U 1636- 53 to be in the range $1.6<M<1.9 \mathrm{M}_{\odot}$.

In addition, as can be seen in the two panels of Fig. 3, not all choices of pairs of peaks can match the observed linear relation between the observed frequencies. In fact, assuming the mass of the NS to be in the range given by Casares et al. (2006), i.e. $M=1.7 \mathrm{M}_{\odot}$, no choice can match the observational relation except the pair of peaks $o_{1}$ and $o_{3}$ (green diamonds) and $o_{2}$ and $f+o_{1}$ (blue squares), but only when the NS is assumed to have a mass $M=1.4 \mathrm{M}_{\odot}$; even

Table 2. Ratio between the frequencies of the selected modes measured from the simulations. Each value reports the ratio between the frequency of the mode shown horizontally with the frequency of the mode shown vertically. Shown in boldface in the bottom right corner of the Table is the ratio of the frequencies of the upper and lower $\mathrm{kHz} \mathrm{QPOs}, v_{u} / \nu_{l}$, for the source NS LMXB 4U 1636 - 53.

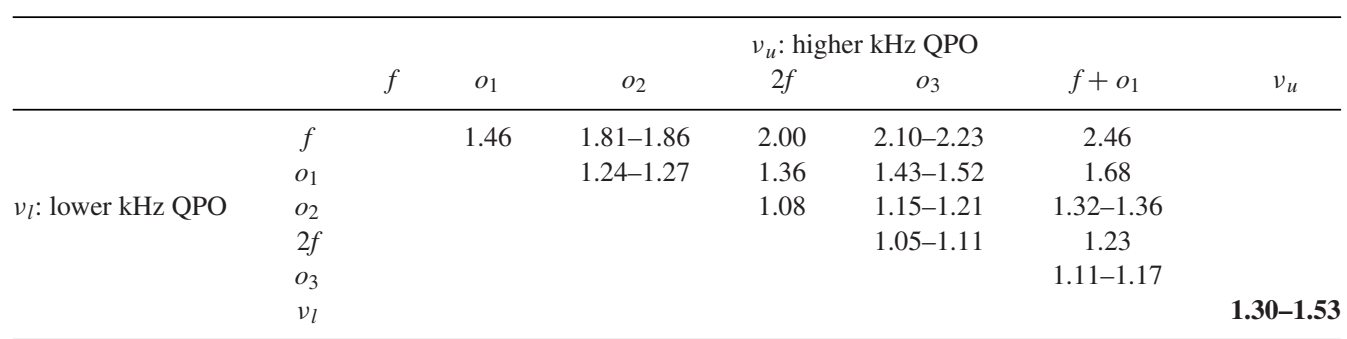



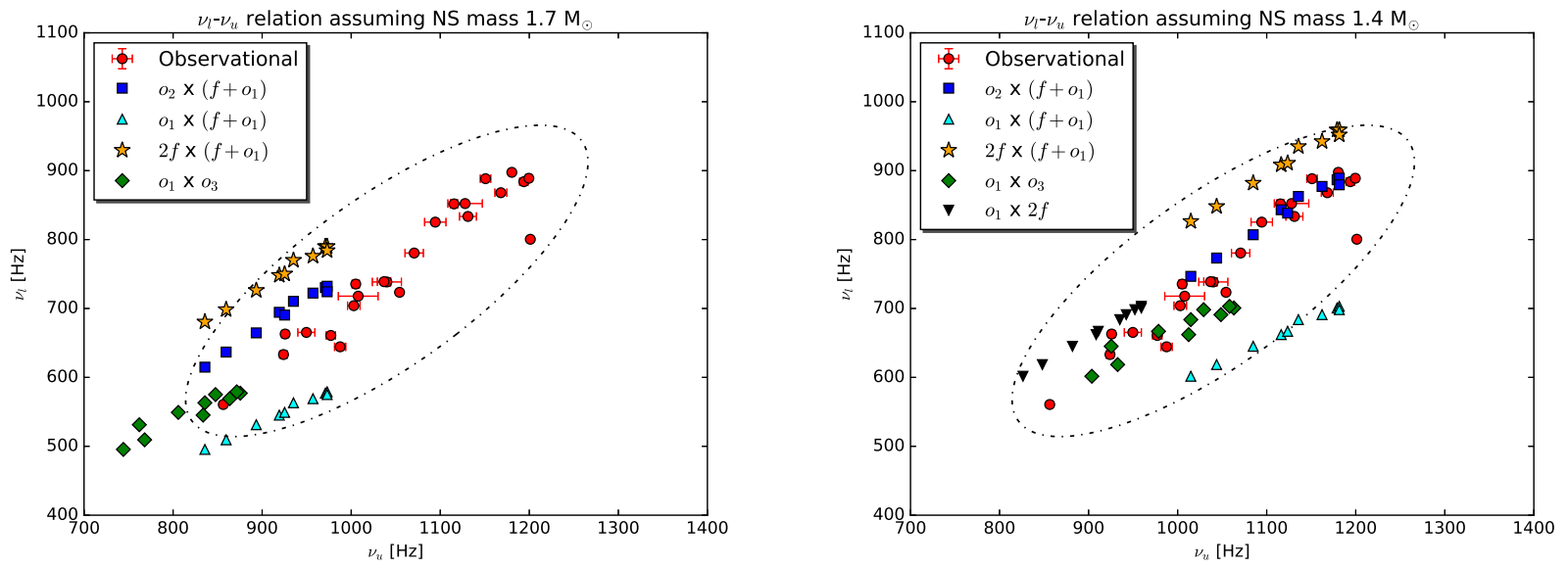

Figure 3. Selected pairs of peaks in the PSD whose numerical relations are presented in Table 2. A larger torus gives rise to lower characteristic frequencies, whereas higher masses for the central star result in lower frequencies. The lower the value of the NS mass, the higher the values of the frequencies of the peaks. The frequencies of the simulated peaks are scalable with the mass of the NS, $v_{1} / v_{2}=M_{2} / M_{1}$, as are the peak separations $\Delta v_{1} / \Delta v_{2}=M_{2} / M_{1}$. The dot-dashed ellipse delineates a region around the observational relation where suitable choices of pairs of peaks are found.

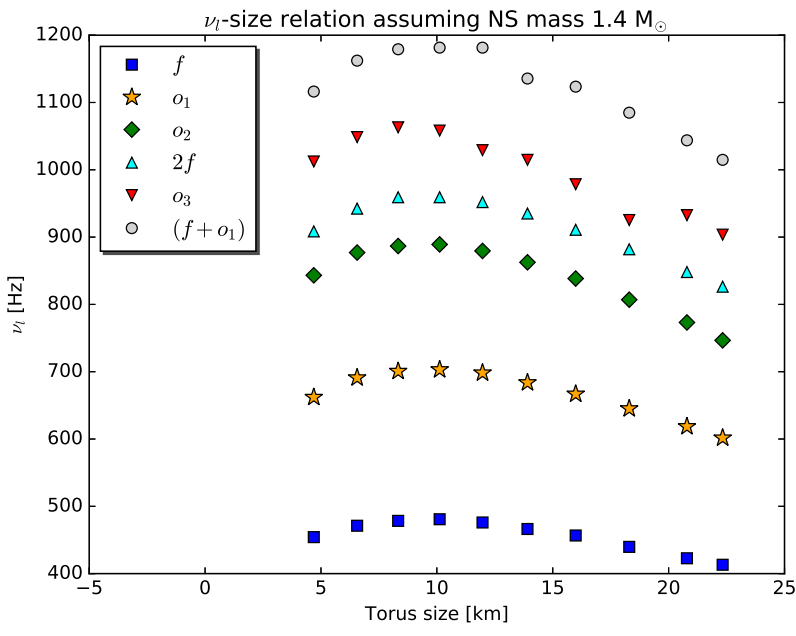

Figure 4. Frequency-size relation for a NS of mass $M=1.4 \mathrm{M}_{\odot}$ and where different colours refer to different oscillation modes. Note that there is a general non-linear behaviour yielding larger frequencies for smaller tori. Notice that the sizes also scale by the ratio of masses. The sizes in the horizontal axis of this figure are the ones from Table 1 times 1.4/1.7.

in these cases, however, the match is marginal. More specifically, in the case of peaks $o_{1}$ and $o_{3}$, although the slope of the correlation and the peak separation seem to be in agreement with the observational relation, the smallest physically meaningful torus, which displays the highest frequencies in this sequence, only reaches the lowest frequencies of the observational relation (see left-hand panel of Fig. 3). In the opposite way, the modes $o_{2}$ and $f+o_{1}$, although promising since they match the observed relation for high frequencies, i.e. for $\left[v_{o_{2}}, v_{f+o_{1}}\right]>[740,1010] \mathrm{Hz}$, do not extend to the low-frequency end of the observational relation.

Before concluding this section a few considerations are worth making. First, since the constant $-\ell$ torus size is delimited from above by the requirement that it does not penetrate the NS surface (thus delimiting the frequencies from below), and the oscillation frequencies achieve their maximum at finite torus extent, the range of achievable frequencies is fixed by the model (recall what we said at the end of Section 2.1).
Secondly, we recall that we have here used the SLy4 equation of state, for which a non-rotating star with mass $M=1.7 \mathrm{M}_{\odot}$ has a radius of $11.42 \mathrm{~km}$. On the other hand, if the star is assumed to have a mass $M=1.4 \mathrm{M}_{\odot}$, the radius is $11.67 \mathrm{~km}$, thus restricting the range of sizes of the possible tori. Hence, the equation of state chosen for the NS does play a role in the sense that it defines, through the radius of the star, the boundary of our simulations as well as the maximum size of the torus. Indeed, regarding the $1.4 \mathrm{M}_{\odot}$ case, it would require building a torus bigger than the ones considered so far. Opposite considerations apply for the mode peaks $o_{1}$ and $2 f$ (black inverted triangles) and $o_{1}$ and $o_{3}$ (green diamonds). Both pairs match the low-frequency tail of the observed linear correlation when $M=1.4 \mathrm{M}_{\odot}$, but not the HF end. Going to smaller NS masses would not help much in this case, since to increase the frequencies one would need to reduce the torus' size by reducing the value of the specific angular momentum, which is however very close to the minimum attainable i.e. $\ell \simeq 3.67$ (Rezzolla $\&$ Zanotti 2013); in fact, as remarked above, models smaller than Tor .03 would be difficult to justify physically because of their small size (cf. Table 1).

Thirdly, although the oscillation modes found do not match exactly the entire observed $\mathrm{kHz}$ QPOs frequency relation, this approximation provides more information than was previously expected. We recall that the observed $v_{l}-v_{u}$ relation, which is detectable when both the lower and the upper $\mathrm{kHz}$ QPOs appear, is thought to arise from the rapidly changing environment around the NS. Indeed, the appearance of both $\mathrm{kHz}$ QPOs occurs only in the left-hand lower corner of the CCD, which is also the region of the CCD where the system changes considerably (Zhang et al. 2017). We show in Fig. 5 the CCD for $4 \mathrm{U}$ 1636-36, together with the corresponding X-ray luminosity as indicated by the colour code in units of the Eddington luminosity. Recalling that the CCD depends on the definition of the hard and soft colours, and following Altamirano et al. (2008), we define the 'hard colour' (HC) as the 9.7-16.0 keV/6.0-9.7 keV count rate ratio and the 'soft colour' (SC) as the $3.5-6.0 \mathrm{keV} / 2.0$ $3.5 \mathrm{keV}$ count rate ratio. Note that the $\mathrm{X}$-ray luminosity is higher in the region of the CCD where both $\mathrm{kHz}$ QPOs are simultaneously detected, thus enabling the detection $v_{l}-v_{u}$ observational relation (see Zhang et al. 2017, and references therein for more details). Since the $p$-mode frequencies of the tori are in first approximation related to their size, the sequence of tori considered here could represent different states of $4 \mathrm{U} 1636-53$. More specifically, it is plausible 


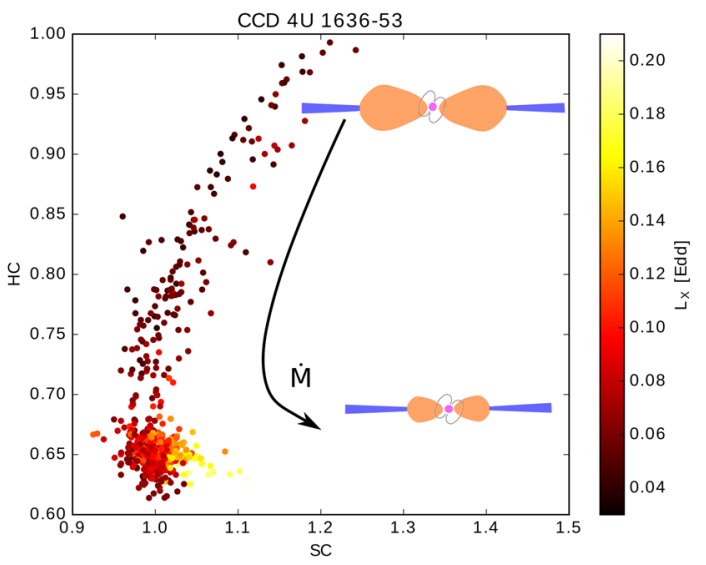

Figure 5. $\mathrm{CCD}$, where the horizontal and vertical axes represent the 'hard colour', HC, and the 'soft colour', SC, respectively. (see main text for details) Larger tori show lower frequencies, in general, and should be placed in the upper parts of the $\mathrm{CCD}$, where the frequencies are, in general, lower, especially for the upper $\mathrm{kHz} \mathrm{QPO}$. Also shown schematically is the direction of increase of the mass-accretion rate and a cartoon showing two suggested states of the accretion disc: In the upper part, the mass-accretion rate is low and disc is farther away from the central object, allowing a bigger torus and lower QPOs. Conversely, in the lower part, the accretion rate is high and the disc is closer to the central object, compressing the torus and yielding higher QPOs. This framework can be used to explain both the observations and the results of the simulations (see main text for details).

to imagine that an external mechanism, possibly within the accretion flow, could determine the size of the torus at a given moment as the source moves across the CCD, perturbing the torus in the process.

Since there is an anticorrelation between the frequencies of the QPOs and the hard colour for Atoll sources (Linares 2009), specially for the upper $\mathrm{kHz}$ QPOs, ${ }^{4}$ it is then tempting to place the tori in the CCD according to their sizes, and we envisage that larger tori are placed in the upper right-hand corner of the CCD (when the source is in the hard state) and smaller tori are placed in the lower left-hand corner of the CCD (when the source is in the soft state) as depicted schematically in Fig. 5. We conjecture, therefore, that the variation of the X-ray luminosity across the CCD is responsible, through the accretion rate, for changes in size of the torus around the NS. This idea is reported schematically using the data of $4 \mathrm{U} 1636-53$ also in Fig. 5: as the accretion rate increases from hard to soft states (see, for example, Liu, Meyer \& Meyer-Hofmeister 2005; Done, Gierliński \& Kubota 2007, and references therein), so does the $\mathrm{X}$-ray luminosity and the accretion disc extend closer to the surface of the NS. Consequently, the overall observational picture seems to be consistent with placing large tori in hard states - when the disc is expected to be more distant and the tori can 'expand' and small tori in soft states - when the disc is 'compressed' as a result of the increased accretion rate (see also Esin, McClintock \& Narayan 1997; Fragile et al. 2016).

Fourthly, we can compare and contrast our results with those obtained recently by other groups. In particular, we note that our results are in agreement with those of Török et al. (2016), where the authors employ a similar idea, but consider non-axisymmetric frequencies. Their work is based on the analysis by Straub \& Šrámková (2009), who derived pressure-corrected fully general-relativistic expressions for the eigenfunctions and eigenfrequencies of the radial and vertical epicyclic modes of a slightly non-slender, constant specific angular momentum torus. Török et al. (2016) built a sequence of tori with different thicknesses, and for each model, they found the modes of oscillation, identifying the lower and upper $\mathrm{kHz}$ QPOs with the frequency of the non-axisymmetric $m=-1$ radial epicyclic mode and the Keplerian frequency at the centre of the torus, respectively. In this way, they were able to match the $v_{l}-v_{u}$ relation for the $\mathrm{kHz}$ QPOs considerably better than previous models, such as the relativistic-precession model (Stella \& Vietri 1999), constraining the mass of the NS in $4 \mathrm{U} 1636-53$ to be $M=1.69 \mathrm{M}_{\odot}$. More recently, Parthasarathy, Kluźniak \& Čemeljić (2017) performed axisymmetric ideal-MHD Newtonian simulations of oscillating cuspfilling tori orbiting a non-rotating NS. They followed the response of the mass-accretion rate to the oscillations of the torus and related this to the boundary layer formed at the surface of the NS, finding that the most prominent mode of oscillation in the massaccretion rate is the radial epicyclic mode, which they associated with the lower $\mathrm{kHz}$ QPO; they could not detect, however, the upper $\mathrm{kHz}$ QPO. We note therefore the analogy with our suggestion of searching an imprint of the torus' oscillations on the mass-accretion rate, which is ultimately related to the boundary-layer luminosity, as demonstrated by Gilfanov, Revnivtsev \& Molkov (2003) and Gilfanov \& Revnivtsev (2005).

Fifthly, Mishra et al. (2017) simulated 22 hydrodynamic tori and found that radial velocity perturbations excite the radial and plus modes, whereas vertical velocity perturbations trigger the vertical and X modes. 'Diagonal' velocity perturbations, like the one we used here, excite a combination of all. They also detected the breathing mode. Here, the $f$ mode is identified with the radial mode, whereas the $o_{1}$ mode corresponds to the plus mode. ${ }^{5}$ In our work, however, the vertical, $\mathrm{X}$, and breathing modes could not be identified, or because they are out of the frequency range we are looking at or because the power of the corresponding peaks are very small and our method discarded them. A difference between the simulations performed by Mishra et al. (2017) and ours is that their tori are much more slender than ours and that is why their frequencies match the analytical ones (see expressions 10 to 14 in their paper) for the slender tori, as depicted in their figs 6,7 , and 8 .

Finally, recent observational and theoretical advances have demonstrated a fairly robust connection between the high frequency QPOs (HFQPOs) and the type-C QPOs in BH LMXBs (Motta et al. 2014, 2015; Fragile et al. 2016). For NS LMXB Z sources, the association was previously known between the horizontal branch oscillations (HBOs) and the type-C QPOs from BH LMXBs (Casella, Belloni \& Stella 2005). Motta et al. (2017) extended this association to the HBO-like QPOs from NS LMXB atoll sources, although less clearly. In the context of the possible correlation between $\mathrm{HBO} / \mathrm{HBO}-$ like QPOs and the $\mathrm{kHz}$ QPOs in NS LMXBs, our model can also be extended, via longer simulations in order to improve the frequency resolution, to test these correlations. If confirmed by observations and simulations, this would be very constraining to any QPO model.

\footnotetext{
${ }^{4}$ In $4 \mathrm{U} 1636-53$, the upper QPO varies from $\sim 400 \mathrm{~Hz}$ at $\mathrm{HC}=1.0$ to $\sim 1220 \mathrm{~Hz}$ at $\mathrm{HC}=0.65$; the lower $\mathrm{QPO}$ is detected at $\sim 550 \mathrm{~Hz}$ for $(\mathrm{HC}$, $\mathrm{SC})=(0.7,0.98)$ and at $\sim 890 \mathrm{~Hz}$ at $(\mathrm{HC}, \mathrm{SC})=(0.62,1.1)$.
} ${ }^{5}$ Confirmed by calculations performed by Dr Odele Straub (private com-
munications). 


\section{CONCLUSIONS}

We have performed general-relativistic simulations of non-selfgravitating and axisymmetric tori with constant specific angular momentum orbiting around a non-rotating NS. After triggering oscillations via a small radial and vertical velocity perturbation, we have followed their evolution and setup a precise scheme for the robust identification of the modes in the PSDs. In agreement with the linear analysis by Rezzolla et al. (2003b), Zanotti et al. (2003), and Zanotti et al. (2005), we have identified the fundamental $p$-mode frequency and its overtones, thus determining eight different frequencies of increasing value in the PSD of various hydrodynamical quantities, such as the rest-mass density. The peaks correspond to the modes of oscillation: $f, o_{1}, o_{2}, 2 f, o_{3}, f+o_{1}, f+o_{2}$, and $3 f$, as marked in the classification of Zanotti et al. (2005). Also in agreement with previous work on tori around $\mathrm{BHs}$, we have found that the value of the fundamental frequency (and hence of the overtones) is inversely proportional to the torus size, scaling linearly with the mass of the star. The dependence of the frequencies on the size of the torus for a given NS mass was then used to compare with the observational relation between the upper and the lower $\mathrm{kHz}$ QPOs in NS LMXB 4U 1636 - 53. This was done by appropriately selecting pairs of peaks in the PSDs and relating each pair to the lower and upper $\mathrm{kHz}$ QPOs, $v_{l}, v_{u}$, with the goal of reproducing the observed linear relation.

Using the ten simulated models and taking into account all possible mode pairs, we have found that as long as we restrict our analysis to tori with constant specific-angular momentum, it is not possible to match the entire observational $v_{l}-v_{u}$ relation, although there are pairs of modes which either reproduce well the observed relation, e.g. modes $o_{2}$ and $f+o_{1}$, but in a limited frequency range, or have correct linear slope, e.g. modes $o_{1}$ and $o_{3}$, but that require stellar masses $M \simeq 1.4 \mathrm{M}_{\odot}$ and hence smaller than what expected from other analyses. Overall, while our simulations provide promising evidence that the phenomenology observed in 4U $1636-53$ could be explained in terms of the $p$-mode oscillations of tori around the $\mathrm{NS}$, it is also clear that the torus models considered here with a constant specific angular momentum can only provide a marginal match with the observed data. This is essentially due to the limitations set by the interplay between the torus size and the radius of the star.

These considerations promote at least three different directions in which our analysis can be improved and that we will consider in future work. First, we can extend this analysis to more general distributions of the specific angular momentum and determine whether the different tori sizes that will be allowed in this way will also provide a better match to the observations. Secondly, we can reconsider our models using fully three-dimensional simulations that would excite non-axisymmetric modes. It is then possible that, with a larger number of modes available, it will be easier to obtain a more accurate match with the observational data. Finally, we can deepen our astrophysical understanding of LMXBs by modelling the radiative processes thought to occur in these systems and their subsequent emission properties and signatures. In particular, using the results of the simulations coupled with the solution of the general-relativistic radiative transfer problem, we can produce synthetic spectra and light curves where time and phase lags will be an integral part of our modelling. (It was demonstrated by Schnittman \& Rezzolla 2006 that the frequencies are actually translated into the light curves, but with varying strengths.) In this way, we will be able to study other properties of the QPOs, e.g. the fractional amplitude, and correlations with energy and frequency of the QPOs (see de Avellar et al. 2016).

\section{ACKNOWLEDGEMENTS}

MGBA acknowledges the financial support from Fundação de Amparo à Pesquisa do Estado de São Paulo (FAPESP) 2015/20553-0 and from the FAPESP Thematic Project 2013/26258-4. Additional support comes from 'NewCompStar', COST Action MP1304, the LOEWE-Program in HIC for FAIR, the European Union's Horizon 2020 Research and Innovation Programme (Grant 671698) (call FETHPC-1-2014, project ExaHyPE), and the ERC synergy grant 'BlackHoleCam: Imaging the Event Horizon of Black Holes' (Grant No. 610058). MGBdA is also grateful to the Institut für Theoretische Physik for the kind hospitality and to E. Ribeiro, Dr P. Bult, and prof. T. Belloni for the useful comments. MGBdA and the authors specially acknowledge Dr Odele Straub for very useful comments and for helping to identify the frequency modes through pressure corrected calculations and to have noticed a small ordering mistake in previous Fig. 4. ZY acknowledges support from an Alexander von Humboldt Fellowship.

\section{REFERENCES}

Abramowicz M., Jaroszynski M., Sikora M., 1978, A\&A, 63, 221

Abramowicz M. A., Karas V., Kluzniak W., Lee W. H., Rebusco P., 2003, PASJ, 55, 467

Abramowicz M. A., Kluzniak W., Stuchlik Z., Torok G., 2004, preprint (arXiv)

Altamirano D., van der Klis M., Méndez M., Jonker P. G., Klein-Wolt M., Lewin W. H. G., 2008, ApJ, 685, 436

Artigue R., Barret D., Lamb F. K., Lo K. H., Miller M. C., 2013, MNRAS, 433, L64

Bakala P., Goluchová K., Török G., Šrámková E., Abramowicz M. A. Vincent F. H., Mazur G. P., 2015, A\&A, 581, A35

Barret D., Olive J.-F., Miller M. C., 2006, MNRAS, 370, 1140

Belloni T., Méndez M., Homan J., 2005, A\&A, 437, 209

Belloni T., Méndez M., Homan J., 2007, MNRAS, 376, 1133

Blaes O. M., Arras P., Fragile P. C., 2006, MNRAS, 369, 1235

Blaes O. M., Šrámková E., Abramowicz M. A., Kluźniak W., Torkelsson U., 2007, ApJ, 665, 642

Bursa M., Abramowicz M. A., Karas V., Kluźniak W., 2004, ApJ, 617, L45

Casares J., Cornelisse R., Steeghs D., Charles P. A., Hynes R. I., O’Brien K., Strohmayer T. E., 2006, MNRAS, 373, 1235

Casella P., Belloni T., Stella L., 2005, ApJ, 629, 403

Daigne F., Font J. A., 2004, MNRAS, 349, 841

de Avellar M. G. B., Méndez M., Sanna A., Horvath J. E., 2013, MNRAS, 433, 3453

de Avellar M. G. B., Méndez M., Altamirano D., Sanna A., Zhang G., 2016, MNRAS, 461, 79

Di Salvo T., Méndez M., van der Klis M., 2003, A\&A, 406, 177

Done C., Gierliński M., Kubota A., 2007, A\&AR, 15, 1

Douchin F., Haensel P., 2001, A\&A, 380, 151

Esin A. A., McClintock J. E., Narayan R., 1997, ApJ, 489, 865

Fishbone L. G., Moncrief V., 1976, ApJ, 207, 962

Font J. A., Daigne F., 2002, MNRAS, 334, 383

Fragile P. C., Straub O., Blaes O., 2016, MNRAS, 461, 1356

Gilfanov M., Revnivtsev M., 2005, Astron. Nachr., 326, 812

Gilfanov M., Revnivtsev M., Molkov S., 2003, A\&A, 410, 217

Hasinger G., van der Klis M., 1989, A\&A, 225, 79

Kluźniak W., Abramowicz M. A., Kato S., Lee W. H., Stergioulas N., 2004, ApJ, 603, L89

Kozlowski M., Jaroszynski M., Abramowicz M. A., 1978, A\&A, 63, 209

Lee W. H., Abramowicz M. A., Kluźniak W., 2004, ApJ, 603, L93

Linares M., 2009, PhD thesis, Sterrenkundig Instituut 'Anton Pannekoek, Univ. Amsterdam

Lin Y.-F., Boutelier M., Barret D., Zhang S.-N., 2011, ApJ, 726, 74

Liu B. F., Meyer F., Meyer-Hofmeister E., 2005, A\&A, 442, 555

Ludlam R. M. et al., 2017, ApJ, 836, 140 
Lyu M., Méndez M., Altamirano D., 2014, MNRAS, 445, 3659

Mauche C. W., 2002, ApJ, 580, 423

Mazur G. P., Vincent F. H., Johansson M., Šramková E., Török G., Bakala P., Abramowicz M. A., 2013, A\&A, 554, A57

Méndez M., 2006, MNRAS, 371, 1925

Miller M. C., Lamb F. K., Psaltis D., 1998, ApJ, 508, 791

Mishra B., Vincent F. H., Manousakis A., Fragile P. C., Paumard T., Kluźniak W., 2017, MNRAS, 467, 4036

Montero P. J., Zanotti O., 2012, MNRAS, 419, 1507

Montero P. J., Zanotti O., Font J. A., Rezzolla L., 2007, MNRAS, 378, 1101

Montero P. J., Font J. A., Shibata M., 2008, Phys. Rev. D, 78, 064037

Montero P. J., Font J. A., Shibata M., 2010, Phys. Rev. Lett., 104, 191101

Motta S. E., Belloni T. M., Stella L., Muñoz-Darias T., Fender R., 2014, MNRAS, 437, 2554

Motta S. E., Casella P., Henze M., Muñoz-Darias T., Sanna A., Fender R., Belloni T., 2015, MNRAS, 447, 2059

Motta S. E., Rouco-Escorial A., Kuulkers E., Muñoz-Darias T., Sanna A., 2017, MNRAS, 468, 2311

Novikov I. D., Thorne K. S., 1973, in Dewitt C., Dewitt B. S., eds, Black Holes (Les Astres Occlus). Gordon and Breach, Paris, p. 340

Parthasarathy V., Kluźniak W., Čemeljić M., 2017, MNRAS, 470, L34

Porth O., Olivares H., Mizuno Y., Younsi Z., Rezzolla L., Moscibrodzka M., Falcke H., Kramer M., 2017, Comput. Astrophys. Cosmol., 4, 1

Psaltis D., Belloni T., van der Klis M., 1999, ApJ, 520, 262

Reig P., van Straaten S., van der Klis M., 2004, ApJ, 602, 918

Remillard R. A., McClintock J. E., 2006, ARA\&A, 44, 49

Rezzolla L., Zanotti O., 2013, Relativistic Hydrodynamics. Oxford Univ. Press, Oxford

Rezzolla L., Yoshida S., Zanotti O., 2003a, MNRAS, 344, 978

Rezzolla L., Yoshida S., Maccarone T. J., Zanotti O., 2003b, MNRAS, 344, L37
Sanna A., Méndez M., Belloni T., Altamirano D., 2012, MNRAS, 424, 2936

Sanna A., Méndez M., Altamirano D., Belloni T., Hiemstra B., Linares M., 2014, MNRAS, 440, 3275

Schnittman J. D., Rezzolla L., 2006, ApJ, 637, L113

Shakura N. I., Sunyaev R. A., 1973, A\&A, 24, 337

Stella L., Vietri M., 1999, Phys. Rev. Lett., 82, 17

Straub O., Šrámková E., 2009, Class. Quantum Gravity, 26, 055011

Török G., Goluchová K., Horák J., Šrámková E., Urbanec M., Pecháček T., Bakala P., 2016, MNRAS, 457, L19

van der Klis M., 2006, in Lewin W. H. G., van der Klis M., eds, Compact Stellar X-ray Sources. Cambridge Astrophysics Series, No. 39. Cambridge Univ. Press, Cambridge, p. 39

van Straaten S., van der Klis M., di Salvo T., Belloni T., 2002, ApJ, 568, 912

van Straaten S., van der Klis M., Méndez M., 2003, ApJ, 596, 1155

van Straaten S., van der Klis M., Wijnands R., 2005, ApJ, 619, 455

Vincent F. H., Mazur G. P., Straub O., Abramowicz M. A., Kluźniak W., Török G., Bakala P., 2014, A\&A, 563, A109

Warner B., Woudt P. A., 2002, MNRAS, 335, 84

Wijnands R., van der Klis M., 1999, ApJ, 514, 939

Wijnands R. A. D., van der Klis M., van Paradijs J., Lewin W. H. G., Lamb F. K., Vaughan B., Kuulkers E., 1997, ApJ, 479, L141

Zanotti O., Rezzolla L., Font J. A., 2003, MNRAS, 341, 832

Zanotti O., Font J. A., Rezzolla L., Montero P. J., 2005, MNRAS, 356, 1371

Zhang G., Méndez M., Sanna A., Ribeiro E. M., Gelfand J. D., 2017, MNRAS, 465, 5003

This paper has been typeset from a $\mathrm{T}_{\mathrm{E}} \mathrm{X} / \mathrm{LAT} \mathrm{EX}$ file prepared by the author. 